Периоперационное

обезболивание пациентки

с морбидным ожирением

при бариатрической операции. Клиническое наблюдение и обзор литературы

Е.Ю. Сырчин1', Р.Е. Лахин², Э.М. Давлетшина', А.А. Гражданкин ${ }^{1}$

1 ФГБОУВО «Башкирский государственный медицинский университет» МЗ РФ, Уфа, Россия

${ }^{2}$ ФГБВОУ ВО «Военно-медицинская академия имени

С.М. Кирова» МО РФ, Санкт-Петербург, Россия

Peфepam

Обоснование. Анестезиологическое обеспечение пациентов с морбидным ожирением при бариатрических операциях представляет собой ряд проблем, включая «трудный» сосудистый доступ, «трудные» дыхательные пути, повышенный риск аспирации, изменение фармакокинетики анестезиологических препаратов, повышенный риск развития послеоперационной депрессии дыхания, связанной с опиоидами.

Методы. Описание клинического случая. Пациентке 32 лет, рост - 162 см, масса тела - 200 кг (индекс массы

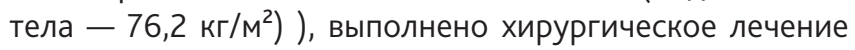
по поводу морбидного ожирения (гастроэнтерошунтирование) с применением сочетанной анестезии (общая анестезия с десфлураном в сочетании с эпидуральной анальгезией ропивакаином 2 мг/мл).

Результаты. Для катетеризации периферической вены и установки эпидурального катетера был использован ультразвуковой контроль. Также в связи с высоким риском «трудных» дыхательных путей (по шкале MOCKBA TD 4 балла) пациентке выполнена фибробронхоскопом оротрахеальная интубация в сознании с седацией дексмедетомидином до уровня -1 по Ричмондской шкале возбуждения-седации. Ранняя активизация пациентки достигалась управляемой анестезией и мультимодальным обезболиванием в послеоперационном периоде. Анальгезия после операции с помощью продленного эпидурального введения 0,2 \% ропивакаина со скоро-

\section{Perioperative pain management of the patient with morbid obesity in bariatric surgery. Clinical report and literature review}

\author{
E.Yu. Syrchin ${ }^{1}$, R.E. Lakhin², E.M. Davletshina',
} A.A. Grazhdankin ${ }^{1}$

\author{
${ }^{1}$ Bashkir State Medical University, Ufa, Russia \\ 2 S.M. Kirov Military Medical Academy, Ministry of Defense \\ of the Russian Federation, Saint Petersburg, Russia
}

\section{Abstract}

Background. Anesthetic management of patients with morbid obesity during bariatric surgery presents a number of problems, including "difficult" vascular access, "difficult" airways, increased risk of aspiration, changes in the pharmacokinetics of anesthetics, and an increased risk of postoperative respiratory depression associated with opioids.

Methods. Case description Patient 32 years old, height $162 \mathrm{~cm}$, body weight $200 \mathrm{~kg}$ (body mass index $76.2 \mathrm{~kg} /$ $\mathrm{m}^{2}$ ) underwent surgical treatment for morbid obesity (gastroentero bypass) using combined anesthesia (general anesthesia with desflurane combined with epidural analgesia ropivacaine $2 \mathrm{mg} / \mathrm{ml}$ ).

Results. Ultrasound navigation was used to catheterize the peripheral vein and insert an epidural catheter. Also, due to the high risk of difficult airways (according to the MOSCOW TD scale -4 points), the patient underwent fiberoptic orotracheal intubation with in consciousness with sedation by dexmedetomidine to level -1 on the Richmond excitation-sedation scale. Early activation of the patient was achieved by controlled anesthesia and multimodal analgesia in the postoperative period. Analgesia after surgery using prolonged epidural administration of $0.2 \%$ ropivacaine at a rate of $4-8 \mathrm{ml} / \mathrm{h}$, intravenous paracetamol 1 gram every 8 hours. 2 hours after the end of the operation, the patient sat down on the bed with her legs down and got up for the first time after 6 hours. On the second day, she was transferred to a specialized department with prolonged epidural analgesia, the duration of which was 72 hours. Complications were not observed in the postoperative period. The patient was discharged from 
стью 4-8 мл/ч и парацетамола внутривенно 1,0 каждые 8 часов. Через 2 часа после окончания операции пациентка села на кровать с опущенными ногами, а впервые встала на ноги через 6 часов. На вторые сутки переведена в профильное отделение с продленной эпидуральной анальгезией, длительность которой составила 72 часа. Осложнений в послеоперационном периоде не наблюдали. Пациентка была выписана из клиники на восьмые сутки после оперативного вмешательства в удовлетворительном состоянии без активных жалоб.

Заключение. В данном клиническом случае выбранная тактика послеоперационного обезболивания позволила достичь хорошей анальгезии, провести раннюю активизацию пациентки и начать процедуры ранней реабилитации.

Ключевые слова: морбидное ожирение, ультразвуковой контроль, анестезия, интубация трахеи, гастроэнтерошунтирование, десфлуран, ропивакаин, эпидуральная, реабилитация

Для корреспонденции: Лахин Роман Евгеньевич - д-р мед. наук, профессор кафедры анестезиологии и реаниматологии ФГБВОУ ВО «Военно-медицинская академия им. С.М. Кирова» МО РФ, Санкт-Петербург; е-mail: doctorlahin@yandex.ru

ए Для цитирования: Сырчин Е.Ю., Лахин Р.Е., Давлетшина Э.М., Гражданкин А.А. Периоперационное обезболивание пациентки с морбидным ожирением при бариатрической операции (клинический случай). Вестник интенсивной терапии им. А.И. Салтанова. 2020;2:146-153.

๘ Поступила: 12.11 .2019

ㅁ Принята к печати: 02.06.2020 the clinic on the eighth day after surgery in a satisfactory condition without active complaints.

Conclusion. In this clinical case, the chosen tactics of postoperative analgesia made it possible to achieve good analgesia, conduct early activation of the patient, and begin early rehabilitation procedures.

Keywords: morbid obesity, ultrasound control, anesthesia, tracheal intubation, gastroentero bypass, desflurane, ropivacaine, epidural, rehabilitation

For correspondence: Roman E. Lakhin - Doctor of Medical Science, Professor, Department of Anesthesiology and Intensive Care Federal State Budgetary Educational Military Institution of Higher Education Military Medical Academy named after S.M. Kirov of the Ministry of Defense of the Russian Federation, Saint Petersburg; e-mail: doctor-lahin@yandex.ru

¿ For citation: Syrchin E.Yu., Lakhin R.E., Davletshina E.M., Grazhdankin A.A. Perioperative pain management of the patient with morbid obesity in bariatric surgery. Clinical report. Annals of Critical Care. 2020;1:146-153.

๘ Received: 12.11 .2019

目 Accepted: 02.06.2020

\section{Введение}

Морбидное ожирение - результат формирования аномальных или чрезмерных жировых отложений, которые могут наносить вред здоровью. Под морбидным ожирением понимают избыточное отложение жировой массы с индексом массы тела (ИМТ) $\geq 40$ кг/ м $^{2}$ или с ИМТ $\geq 35$ кг/м² при наличии серьезных осложнений, связанных с ожирением $[1,2]$. Количество людей с морбидным ожирением растет год от года. Во всем мире в 2016 г. более 1,9 млрд взрослых старше 18 лет (39 \%) имели избыточный вес. Из них свыше 650 млн страдали ожирением. В России в среднем 30 \% лиц трудоспособного возраста страдают ожирением [3]. Несмотря на значительные экономические затраты по профилактике и лечению данного заболевания в мире, летальность в возрасте 20-35 лет с морбидным ожирением превышает среднестатические показатели в 12 раз, в основном от сердечно-сосудистых и респираторных осложнений $[3,4]$. Ожирение и связанные с ним метаболические нарушения являются серьезной проблемой современной медицины, поскольку приводят к развитию целого ряда заболеваний и осложнений. К серьезным осложнениям (ассоциированным заболеваниям), связанным с ожирением, относятся: сахарный диабет 2-го типа (СД 2-го типа) и предиабетические нарушения углеводного обмена; сердечно-сосудистые заболевания; дислипидемия; синдром обструктивного апноэ сна; синдром Пиквика; хроническая обструктивная болезнь легких; нарушения опорно-двигательной системы; злокачественные опухоли отдельных локализаций; неалкогольная жировая болезнь печени; репродуктивные нарушения [1, 3-7].

Анестезиологическое обеспечение пациентов с морбидным ожирением при бариатрических операциях представляет собой ряд проблем, включая «труд- 
ный» сосудистый доступ, «трудные» дыхательные пути, повышенный риск аспирации, изменение фармакокинетики анестезиологических препаратов, повышенный риск развития послеоперационной депрессии дыхания, связанной с опиоидами $[5,8]$. Обезболивание пациента с морбидным ожирением может быть сложной задачей, требующей индивидуального подхода $[5,9,10]$. Регионарные методики обезболивания у таких пациентов в последние годы получили новое развитие из-за внедрения ультразвукового контроля манипуляций [10-12]. Мультимодальные стратегии обезболивания, включающие нейроаксиальные техники обезболивания, могут снизить частоту развития осложнений, особенно респираторных, в раннем послеоперационном периоде и улучшить исходы лечения пациентов с морбидным ожирением $[5,10,11,13-16]$.

В представленном клиническом случае использован мультимодальный подход к периоперационному обезболиванию, направленный на раннюю активизацию пациентки и профилактику респираторных осложнений.

\section{Подходы к периоперационному обезболиванию пациентов с морбидным ожирением}

Главная цель периоперационного ведения - ранняя активизация и реабилитация пациентов для предотвращения послеоперационных осложнений. Эту цель в свою основу положили концепции Fast Track хирургии («быстрый путь в хирургии») и ERAS (early rehabilitation after surgery - ранняя реабилитация после операции). B отношении периоперационного обезболивания эти концепции требуют отказа от «традиционной» премедикации, включения в схемы обезболивания регионарных блокад, использования в ходе анестезии препаратов с быстрым началом и короткой продолжительностью действия, безопиоидной послеоперационной терапии боли $[5,10,11,15-17]$.

Низкие функциональные резервы диктуют необходимость в ограничении использования препаратов, вызывающих длительную седацию и способных угнетать дыхание, поэтому опиоиды в премедикацию не назначают. Непосредственная задача премедикации решается за счет назначения бензодиазепинов или дексмедетомидина, хотя нужно помнить, что у пациентов, страдающих синдромом сонного апноэ или гиповентиляционным синдромом, проведение седации даже бензодиазепинами в палате перед операцией небезопасно в связи возможностью депрессии дыхания [5, 10, 13, 14, 18$]$.

При проведении анестезии у пациентов с морбидным ожирением следует использовать анестетики с низкой растворимостью в крови и низкой липофильностью, поскольку на этапе пробуждения применение анестетиков с высокой липофильностью приводит к за- медленному пробуждению [5, 10, 18-20]. Препараты выбора у таких пациентов - десфлуран и севофлуран. Анестезия десфлураном может оказаться более предпочтительной по сравнению севофлураном, поскольку десфлуран практически не кумулируется в организме независимо от продолжительности наркоза, так как метаболизм этого препарата очень низок и обладает благоприятным профилем пробуждения [5, 18-20]. Именно поэтому десфлуран приобрел популярность в бариатрической хирургии. Для вводного наркоза в современной анестезиологической практике используют пропофол [21]. Следует помнить, что у пациентов с обструктивным сонным апноэ применение пропофола и бензодиазепинов для седации в премедикации связано с риском гипоксемии $[5,18,19,21]$.

Адекватное послеоперационное обезболивание один из важнейших факторов быстрой послеоперационной реабилитации. По современным представлениям о механизмах развития боли, очень большое значение придается мультимодальности (многокомпонентности) в подходах к решению этой проблемы. Большинство авторов считает, что купирование послеоперационной боли необходимо достигать сочетанием регионарных методов анестезии, опиоидов, использованием нестероидных противовоспалительных препаратов и парацетамола. Хотя нужно помнить, что парацетамол возможно применять только при отсутствии у пациента явлений печеночной дисфункции [5, 10, 11, 13, 15-17].

Минимизация использования или даже полный отказ от опиоидов в послеоперационном периоде обусловлены не только депрессией дыхания, но и развитием отрицательных побочных эффектов: тошнота и рвота, кожный зуд, илеус, задержка мочи, седация и связанная с этим гиподинамия и необходимость нахождения пациента в постели. Кроме этого, развитие толерантности с необходимостью увеличения дозы опиоида для достижения эффекта и гиперальгезии в виде увеличения чувствительности к болевой стимуляции требует увеличения количества и кратности применения опиоидов, при этом формируются порочные круги побочных эффектов, ведущих к развитию осложнений и возможности появления наркотической зависимости $[10,13,15,17]$.

Анальгетики не следует вводить внутримышечно, так как попадание иглой в мышечный массив плохо предсказуемо и по внешним ориентирам практически неопределимо, поэтому биодоступность препаратов остается неясной [10].

Послеоперационное обезболивание с помощью опиоидных трансдермальных терапевтических систем пока не нашло широкого применения в бариатрической хирургии, вероятнее всего, по той же причине плохой предсказуемости биодоступности, хотя в других видах хирургии используется с хорошими результатами [10, 22, 23]. 


\section{Описание клинического случая}

В ноябре 2018 г. в отделение хирургии [заслеплено редакцией] обратилась за консультативной помощью пациентка Ж., 31 год, с жалобами на избыточную массу тела, слабость, одышку при малейшей физической нагрузке. Распределение жировых отложений имеет смешанный характер, рост $-162 \mathrm{~cm}$, масса тела -200 кг (ИМТ - 76,2 кг $\left./ \mathrm{M}^{2}\right)$. Курила до 20 сигарет в день. После утренней сигареты появлялся кашель с трудноотделяемой слизистой мокротой. Отмечался незначительный акроцианоз. Сатурация крови при осмотре в положении сидя составляла 89 \%, в положении лежа возникало чувство нехватки воздуха, а сатурация снижалась до $86 \%$. Частота дыхания - 19 в минуту. При аускультации над легкими, на фоне везикулярного дыхания, выслушивались единичные свистящие хрипы. В нижних отделах дыхание было ослаблено. По шкале STOP-Bang - высокий риск обструктивного апноэ [24]. Был установлен синдром Пиквика (имелась дневная сонливость, полицитемия). При осмотре верхних дыхательных путей трудность интубации по шкале МОСКВА TD оценивали в 4 балла [25]. При измерении артериального давления - артериальная гипертензия: 170 и 100 мм рт. ст. Частота сердечных сокращений - 100 ударов в мин соответствовала пульсу. Пациентка нерегулярно принимала каптоприл в дозировке 50 мг два раза в сутки. В лабораторных данных: глюкоза крови $-6,08$ ммоль/л, холестерин $-6,34$ ммоль/л. При ультразвуковом дуплексном сканировании сосудов и вен нижних конечностей - без особенностей, поверхностные и глубокие вены нижних конечностей проходимы. При эхокардиографии камеры сердца не увеличены, гипертрофия миокарда левого желудочка, фракция выброса - 68 \%, конечно-диастолический объем левого желудочка - 108 мл. По данным спирографии было выявлено нарушение дыхательной функции легких обструктивно-рестриктивного типа тяжелой степени: жизненная емкость легких $-2,04$ л (56,81\%); форсированная жизненная емкость легких $-1,77$ л (50,98 \%); объем форсированного выдоха за первую секунду $-1,08$ л (35,52 \%); индекс Р. Тиффно фактический $-60,7$ \% (71,8 \% от должного). Пациентке был установлен основной диагноз: морбидное ожирение (ИМТ > 40 кг $/ \mathrm{m}^{2}$ ); сопутствующий диагноз: хроническая обструктивная болезнь легких, дыхательная недостаточность II степени, гипертоническая болезнь 2-й степени, общий сердечно-сосудистый риск - IV (очень высокий), нарушение толерантности к глюкозе; дислипидемия; синдром Пиквика. Для подготовки пациентки к плановому оперативному вмешательству даны следующие рекомендации: полный отказ от курения; мониторинг уровня глюкозы крови; коррекция респираторной терапии под контролем пульмонолога; коррекция артериальной гипертензии под контролем терапевта (кардиолога); диета для снижения массы тела.
Повторно пациентка обратилась в конце мая 2019 г. Вес пациентки, несмотря на попытку соблюдать диету, не изменился: 200 кг при росте 162 см. Рекомендации специалистов были выполнены частично. Пациентка продолжала курить, но количество потребляемых сигарет за сутки было уменьшено вдвое. При обследовании был уточнен терапевтический диагноз: гипертоническая болезнь II стадии, I степени медикаментозно достигнутая, общий сердечно-сосудистый риск - IV; с сопутствующей патологией: хроническая обструктивная болезнь легких средней степени тяжести, стадия неполной ремиссии, дыхательная недостаточность I-II стадии; синдром Пиквика. Пациентка получала лечение: 1) лозартан 100 мг вечером; 2) индапамид 2,5 мг утром; 3) бисопролол 2,5 мг утром; 4) розувастатин 10 мг вечером; 5) тиотропия бромид 18 мг 1 вдох 1 раз в день. На фоне назначенного лечения отмечала улучшение общего самочувствия, улучшилась переносимость физической нагрузки, уменьшились интенсивность кашля, количество мокроты. Уровень глюкозы в крови перед операцией составлял 5,3 ммоль/л.

Было принято решение о проведении хирургической операции гастроэнтерошунтирования. При осмотре пациентки перед операцией: кожный покров физиологической окраски, дыхание через нос свободное, при аускультации легких - дыхание везикулярное, проводится во все отделы, хрипов нет. Сатурация крови сидя $-98 \%$, лежа - снижалась до $93 \%$ с возникновением чувства нехватки воздуха. Артериальное давление -140 и 80 мм рт. ст., пульс -84 в мин. Учитывая положительную динамику на фоне проводимой терапии, решено провести плановое оперативное вмешательство под сочетанной анестезией: общая комбинированная, ингаляционный компонент десфлураном и эпидуральной анальгезией ропивакаином. Выбор десфлурана по сравнению с севофлураном для анестезии у пациентов с морбидным ожирением позволяет быстрее достичь вербального контакта и выполнить экстубацию трахеи [26]. Физический статус пациентки по классификации ASA (American Society of Anesthesiologists) IV [27].

Премедикация: октреотид 300 мкг внутривенно, дексаметазон 8 мг внутривенно, ранитидин 50 мг внутривенно.

Проведение анестезии. При поступлении в операционную артериальное давление - 140 и 83 мм рт. ст., пульс - 94 в минуту. Под ультразвуковым контролем катетеризирована левая $v$. basilica с первой попытки катетером $18 \mathrm{G}$ (рис. 1).

В положении сидя с помощью ультразвуковой визуализации проведено определение ориентиров нейроаксиальных структур, осуществлена ультразвуковая разметка срединного доступа для катетеризации эпидурального пространства (рис. 2). С соблюдением правил асептики и антисептики проведена пункция и катетери- 


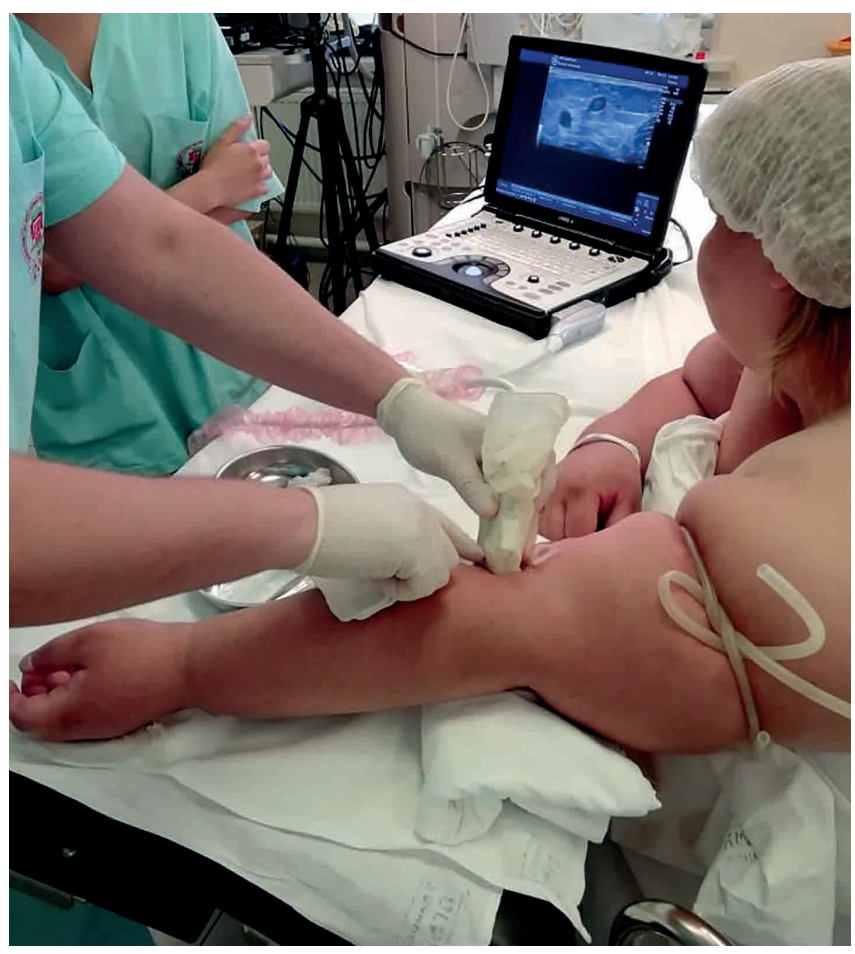

Рис. 1. Обеспечение сосудистого доступа катетеризацией периферической вены под контролем ультразвука

Fig. 1. Providing vascular access by peripheral vein catheterization under ultrasound control

зация эпидурального пространства на уровне Th9-Th10. Глубина залегания эпидурального пространства составила 10 см. Катетер проведен краниально на 6 см. После этого проведена тест-доза 2 \% лидокаином 60 мг. Показатели гемодинамики в течение 5 мин не изменились, признаков спинальной анестезии не было. Для развития эпидуральной анестезии в катетер введено 8 мл 0,5\% раствора ропивакаина.

Учитывая прогнозируемый высокий риск «трудных» дыхательных путей, было принято решение о выполнении фиброоптической интубации трахеи в сознании на фоне сохранения самостоятельного дыхания. Для комфорта пациентки, снижения эмоционального стресса, обеспечения гемодинамической стабильности перед интубацией была выполнена седация дексмедетомидином (0,5 мкг/кг идеальной массы тела). После достижения необходимого уровня седации, позволяющего сохранить сотрудничество пациентки, который составил 1 по Ричмондской шкале возбуждения-седации (RASS - Richmond Agitation-Sedation Scale) [28] на фоне инсуффляции кислородом со скоростью 8 л/мин, выполнена оротрахеальная интубация с помощью фибробронхоскопа (рис. 3).

После интубации произведена индукция в анестезию: внутривенно пропофол 150 мг, фентанил 200 мкг, с целью миоплегии - рокурония бромид 50 мг. Пациентка уложена в HELP (head-elevated laryngoscopy

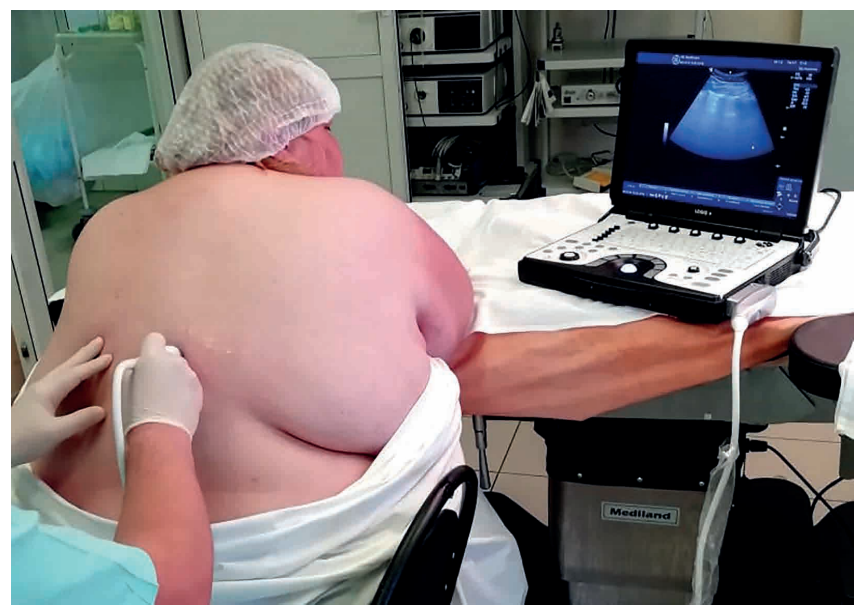

Рис. 2. Ультразвуковая визуализация доступа к эпидуральному пространству

Fig. 2. Ultrasound visualization of access to the epidural space

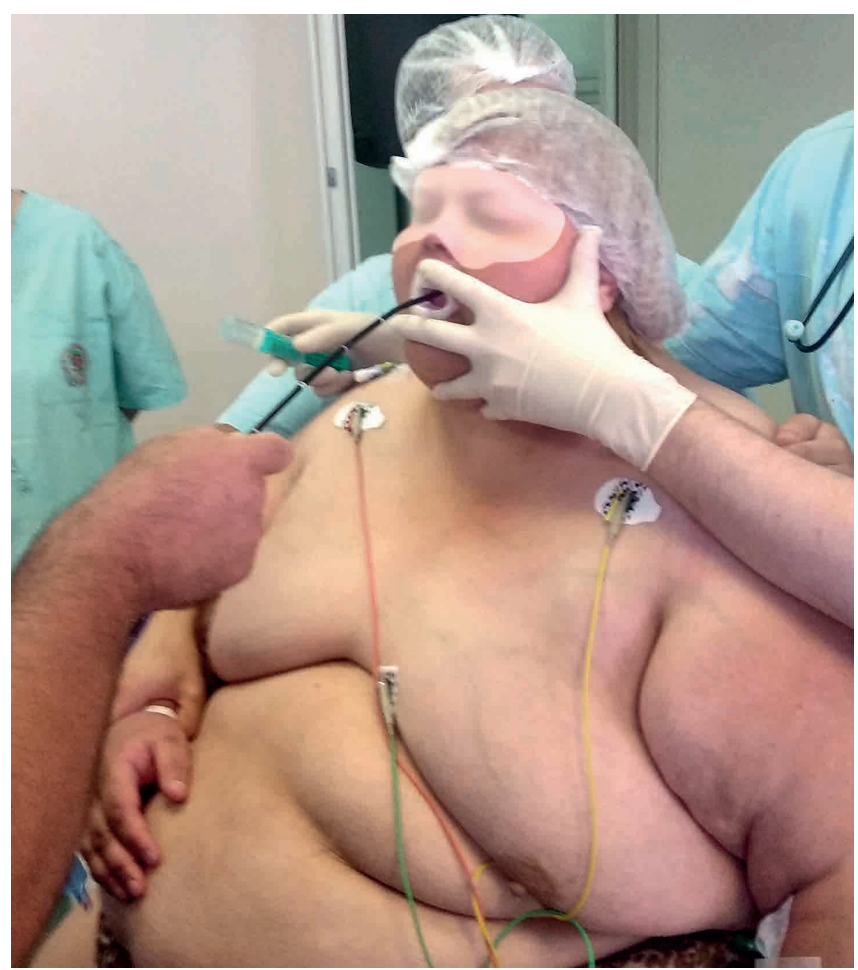

Рис. 3. Фиброоптическая интубация трахеи

Fig. 3. Fibrooptic tracheal intubation

position) - положение на спине с возвышенным головным концом. Перед началом оперативного вмешательства был налажен мониторинг биспектрального индекса (BIS), глубины нейромышечного блока (TOF) в дополнение к стандартному (неинвазивное измерение артериального давления, пульса, сатурации, ЭКГ, температуры тела). Время от поступления пациентки в операционную до начала хирургической операции составило 60 мин. 
Во время оперативного вмешательства поддержание анестезии достигали ингаляцией десфлурана на уровне 0,7-0,9 минимальной альвеолярной концентрации, удерживая показатель BIS в пределах 40-50; анальгезию внутривенным дробным введением фентанила, общий расход которого составил 500 мкг; постоянной инфузией в эпидуральный катетер с помощью шприцевого инфузамата $0,2 \%$ раствора ропивакаина со скоростью 8-10 мл/ч. Миорелаксацию поддерживали введением рокурония бромида под контролем TOF-мониторинга, общий расход составил 100 мг. Респираторная поддержка осуществлялась аппаратом Drager Primus в режиме принудительной вентиляции по объему с положительным давлением в конце выдоха 12-14 см вод. ст. Концентрация кислорода на вдохе поддерживалась на уровне 70-80 \%. Поток свежего газа составил 2 л/мин. Частота аппаратного дыхания подбиралась по концентрации углекислого газа на выдохе. Продолжительность оперативного вмешательства составила 180 мин. Интраоперационная кровопотеря 200 мл, инфузионная терапия - раствор стерофундин изотонический 1000 мл, мочи за время операции получено 200 мл светло-желтого цвета.

Для реверсии остаточного действия миорелаксанта внутривенно использован сугаммадекс 200 мг при показателях TOF 75 \%. Пациентка экстубирована в операционной при показателях TOF 99 \% и BIS 90 \% (рис. 4).

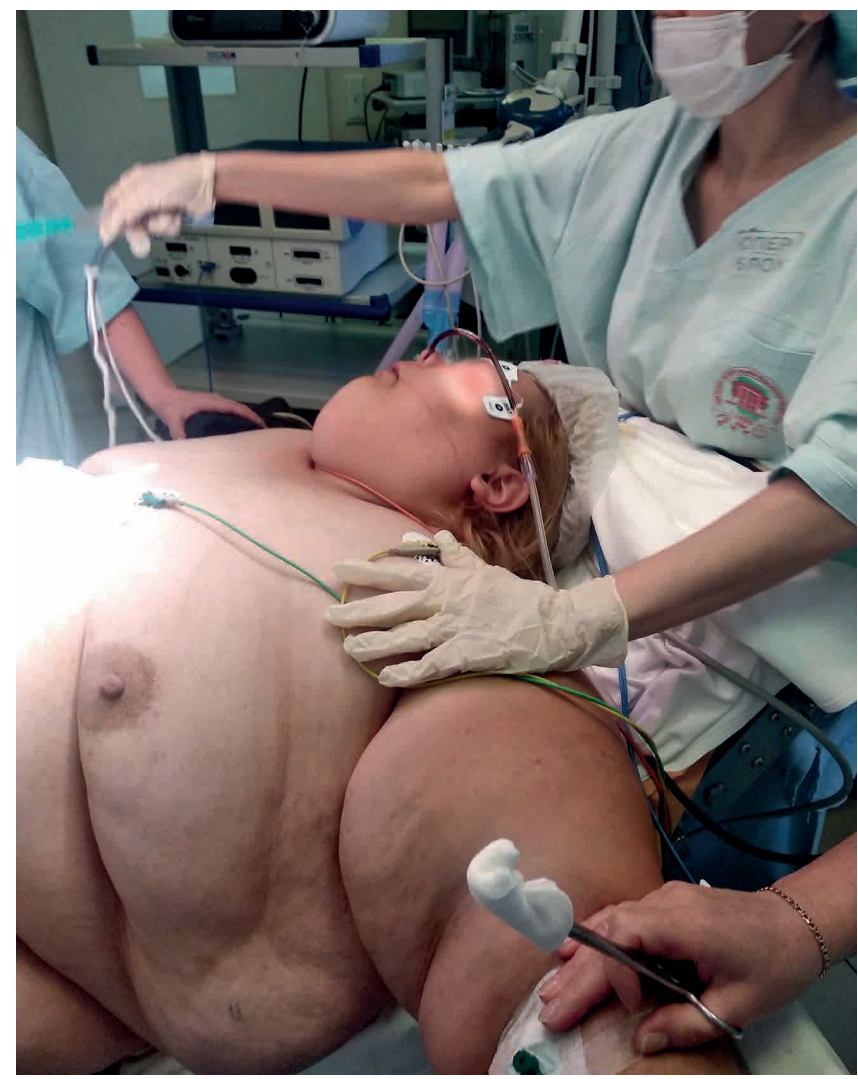

Рис. 4. Экстубация на операционном столе

Fig. 4. Extubation on the operating table
После экстубации пациентка самостоятельно переместилась на многофункциональную реанимационную кровать. При контроле болевого синдрома сразу после перекладывания по цифровой рейтинговой шкале уровень боли составил 2 балла.

В послеоперационном периоде продолжено продленное эпидуральное введение $0,2 \%$ ропивакаина со скоростью 4-8 мл/ч, а также парацетамол внутривенно 1 г каждые 8 часов. На фоне этой противоболевой терапии уровень боли не превышал 2-3 баллов по цифровой рейтинговой шкале даже при движении и кашле. Через 2 часа после окончания операции пациентка села на кровать с опущенными ногами, а впервые встала на ноги через 6 часов. В первые сутки после оперативного вмешательства для профилактики респираторных осложнений проводили 2 сеанса неинвазивной вентиляции легких на протяжении 2 часов каждый аппаратом «Зислайн» с положительным давлением в конце выдоха 8 см вод. ст. Сатурация кислорода при этом возрастала с 92-94\% до 98 \% в положении лежа, с возвышенным головным концом. Пациентка переведена в профильное отделение на вторые сутки с продленной эпидуральной анальгезией, которая продолжалась 72 часа. Осложнений в послеоперационном периоде не наблюдали. Пациентка была выписана из клиники на восьмые сутки после оперативного вмешательства.

\section{Обсуждение}

Периоперационное ведение пациентов с морбидным ожирением всегда сопровождается комплексом проблем, требующих внимания на всех этапах [29]. Вопросы компенсации патологии сердечно-сосудистой и дыхательной систем перед операцией, «трудный» сосудистый доступ, «трудные» дыхательные пути, изменение фармакокинетики анестезиологических препаратов во время операции и анестезии $[4,5,10,15,18,19]$. Перевод пациента с морбидным ожирением на самостоятельное дыхание, повышенный риск развития послеоперационной депрессии дыхания, связанной с опиоидами, обструктивным апноэ, развитие осложнений в виде пневмонии, особенно на фоне застойных изменений в легких, усугубление расстройств сердечно-сосудистой системы, диабета и других $[5,8,10,13,16,18,19]$.

Выполнение инвазивных манипуляций под ультразвуковым контролем позволило повысить их успешность и снизить количество осложнений $[12,30,31]$. В представленном клиническом случае с помощью ультразвука была катетеризирована периферическая вена, пункция которой по внешним ориентирам была бы невозможна. При необходимости анестезиологическая бригада была готова выполнить катетеризацию внутренней яремной вены, предварительный осмотр которой также был проведен. Катетеризация эпиду- 
рального пространства у пациентов с морбидным ожирением также является технически сложной манипуляцией $[9,10,11,19,31]$. Большой слой подкожного жира, затрудняющий пальпацию, несоответствие внешних ориентиров и отростков позвоночника, высокая глубина залегания твердой мозговой оболочки - все это требует ультразвукового контроля для успешного выполнения манипуляции [12, 19]. Мы выполнили ультразвуковую разметку, определив точку вкола, угол продвижения иглы и глубину нахождения твердой мозговой оболочки. Поскольку глубина расположения твердой мозговой оболочки составила $10 \mathrm{~cm}$, была подобрана эпидуральная игла длиной 12 см. Пункцию эпидурального пространства проводили методикой потери сопротивления. Кроме сложности манипуляции, особенностью эпидуральной анестезии у пациентов с морбидным ожирением является более высокая частота развития артериальной гипотензии [9, 31]. Поэтому после нагрузочной дозировки мы приняли решение о поддержании анестезии низкоконцентрированным раствором местного анестетика - 0,2\% раствором ропивакаина. В представленном клиническом случае постоянная инфузия ропивакаина в эпидуральное пространство не привела к развитию артериальной гипотензии, но позволила добиться как в раннем, так и в позднем послеоперационном периоде хорошей анальгезии и обойтись без наркотических анальгетиков. Качественное обезболивание стало основой ранней активизации пациентки и ее быстрой реабилитации по принципам Fast Track хирургии и $\operatorname{ERAS~[5,15-17].~Мы~считаем,~что~именно~адекватная~}$ мультимодальная анальгезия в послеоперационном периоде и подготовка на догоспитальном этапе к ранним реабилитационным процедурам после операции позволили быстро активизировать пациентку и избежать осложнений, в первую очередь связанных с гиподинамией.

\section{Заключение}

Регионарная анестезия, популяризированная для Fast Track и ERAS хирургии, не является рутинной у пациентов с морбидным ожирением. Трудности выполнения манипуляции, возможные осложнения заставляют работать по принципам индивидуального подхода. Ультразвуковые технологии расширили возможности анестезиологов-реаниматологов, предоставив возможность выполнять блокады в тех случаях, в которых стандартные методики блокад по внешним ориентирам становятся невозможными. В данном клиническом случае выбранная тактика послеоперационного обезболивания позволила достичь хорошей анальгезии, провести быструю активизацию пациентки и начать процедуры ранней реабилитации.

Конфликт интересов. Авторы заявляют об отсутствии конфликта интересов.

Информация об информированном согласии. Информированное согласие пациентки на публикацию случая ее болезни получено.

Вклад авторов. Сырчин Е.Ю., Лахин Р.Е., Давлетшина Э.М., Гражданкин А.А. - разработка концепции статьи, получение и анализ фактических данных, написание и редактирование текста статьи, проверка и утверждение текста статьи.

\section{ORCID авторов}

Сырчин Е.Ю. - 0000-0002-0027-6491

Лахин Р.Е. - 0000-0001-6819-9691

Давлетшина Э.М. - 0000-0002-8393-3919

Гражданкин А.А. - 0000-0002-5874-8543

\section{Литература/References}

[1] Дедов И.И., Мельниченко Г.А., Шестакова М.В. и др. Лечение морбидного ожирения у взрослых. Ожирение и метаболизм. 2018; 15(1): 53-70. DOI: 10.14341/OMET2018153-70. [Dedov I.I., Melnichenko G.A., Shestakova M.V., et al. Morbid obesity treatment in adults. Obesity and metabolism. 2018; 15(1): 5370. (In Russ)]

[2] World Health Organization. Obesity: preventing and managing the global epidemic. 1997, Geneva: WHO [Internet] Available from: http://www.who.int/nutrition/publications/obesity/ WHO_ TRS_894/en/ (accessed 15.12.2019).

[3] World Health Organization. Obesity and overweight. Fact sheets [Internet] Available from: https://www.who.int/news-room/factsheets/detail/obesity-and-overweight (accessed 15.12.2019).

[4] Степанова Е.В., Лоранская И.Д., Ракитская Л.Г., Мамедова Л.Д. Ожирение как универсальный фактор риска серьезных заболеваний. Эффективная фармакотерапия. 2019; 15(18):
68-77. DOI: 10.33978/2307-3586-2019-15-18-68-77. [Stepanova Ye.V., Loranskaya I.D., Rakitskaya L.G., Mamedova L.D. Obesity as the Omni-Factor for Serious Diseases. Effective Pharmacotherapy. 2019; 15(18): 68-77. (In Russ)]

[5] Заболотских И.Б., Лебединский К.М., Анисимов М.А. и др. Периоперационное ведение больных с сопутствующим морбидным ожирением (второй пересмотр). Клинические рекомендации. Тольяттинский медицинский консилиум. 2016; 5-6: 38-56. [Zabolotskikh I.B., Lebedinskii K.M., Anisimov M.A., et al. Perioperative management in morbid obesity patients (second revision). Clinical recommendations. Togliatti medical Council. 2016; 5-6: 38-56. (In Russ)]

[6] Guh D.P., Zhang W., Bansback N., et al. The incidence of comorbidities related to obesity and overweight: $A$ systematic review and metaanalysis. BMC Public Health. 2009; 9(1). DOI: 10.1186/1471-2458-9-88. 
[7] Lenz M., Richter T., Muhlhauser I. The morbidity and mortality associated with overweight and obesity in adulthood: a systematic review. Dtsch Arztebl Int. 2009; 106(40): 641-648. DOI: 10.3238/ arztebl. 2009.0641.

[8] De JongA., VerzilliD., Geniez M., et al. Why is the morbidly obese patient at high risk of anesthetic complications? Presse Med. 2018; 47(5): 453-463. DOI: 10.1016/j.lpm.2018.01.016

[9] Ingrande J., Brodsky J.B., Lemmens H.J. Regional anesthesia and obesity. Curr Opin Anaesthesiol. 2009; 22(5): 683-686. DOI: 10.1097/ ACO.0b013e32832eb7bd

[10] Эпштейн С.Л. Периоперационное анестезиологическое обеспечение больных с морбидным ожирением. Регионарная анестезия и лечение острой боли. 2012; 6(3): 5-27. [Epshtein S.L. Perioperative anesthetic management in morbidly obese patients. Regional anesthesia and treatment of acute pain. 2012; 6(3): 5-27. (In Russ)]

[11] Неймарк М.И., Киселев Р.В., Пантюшин А.А. Сравнительная оценка вариантов сочетанной анестезии с использованием ингаляционных анестетиков при хирургическом лечении морбидного ожирения. Вестник анестезиологии и реаниматологии. 2015; 12(1): 17-24. [Neimark M.I., Kiselev R.V., Pantyushin A.A. Comparative assessment of concomitant inhalational anesthesia modes in the surgical treatment of morbid obesity. Bulletin of Anesthesiology and Intensive Care. 2015; 12(1): 17-24. (In Russ)]

[12] Shaylor R., Saifi F., Davidson E., Weiniger C.F. High success rates using ultrasound for neuraxial block in obese patients. Isr Med Assoc J. 2016 18(1): 36-39.

[13] Неймарк М.И., Киселев Р.В. Мультимодальная анальгезия в бариатрической хирургии Регионарная анестезия и лечение острой боли. 2016; 10(4): 254-261. [Neimark M.I., Kiselev R.V. Multimodal analgesia in bariatric surgery. Regional anesthesia and treatment of acute pain. 2016; 10(4): 254-261. (In Russ)]

[14] de Raaff C.A.L., Gorter-Stam M.A.W., de Vries N., et al. Perioperative management of obstructive sleep apnea in bariatric surgery: a consensus guideline. Surg Obes Relat Dis. 2017; 13(7): 1095-1109. DOI: 10.1016/j.soard.2017.03.022

[15] Belcaid l., Eipe N. Perioperative Pain Management in Morbid Obesity Drugs. 2019; 79(11): 1163-1175. DOI: 10.1007/s40265-019-01156-3

[16] Budiansky A.S., Margarson M.P., Eipe N. Acute pain management in morbid obesity - an evidence based clinical update. Surg Obes Relat Dis. 2017; 13(3): 523-532. DOI: 10.1016/j.soard.2016.09.013

[17] Агеенко А.М., Садовой М.А., Шелякина О.В., ОвтинМ.А. Технология ускоренной реабилитации после эндопротезирования тазобедренного и коленного суставов (обзор литературы) Травматология и ортопедия России. 2017; 23(4): 146-155. [Ageenko A.M., Sadovoy M.A., Shelyakina O.V., Ovtin M.A. Fast-track Hip and Knee Arthroplasty (Literature Review). Travmatologiya i ortopediya Rossii. 2017; 23(4): 146-155. (In Russ)] DOI: 10.21823/2311-29052017-23-4-146-155

[18] Bazurro S., Ball L., Pelosi P. Perioperative management of obese patient. Curr Opin Crit Care. 2018, 24(6): 560-567. DOI: 10.1097/ MCC.0000000000000555

[19] Lang L.H., Parekh K., Tsui B.Y.K., Maze M. Perioperative management of the obese surgical patient. Br Med Bull. 2017; 124(1): 135-155 DOI: $10.1093 / \mathrm{bmb} / \mathrm{ldx} 041$
[20] Juvin P., Vadam C., Malek L., et al. Postoperative recovery after desflurane, propofol, or isoflurane anesthesia among morbidly obese patients: a prospective, randomized study. Anesth. Analg. 2000; 91 714-719.

[21] Gaszyński T., Wieczorek A. A comparison of BIS recordings during propofol-based total intravenous anaesthesia and sevoflurane-based inhalational anaesthesia in obese patients. Anaesthesiol Intensive Ther. 2016; 48(4): 239-247

[22] Bakeer A.H., Abdallah N.M. Transdermal fentanyl as an adjuvant to paravertebral block for pain control after breast cancer surgery: A randomized, double-blind controlled trial. Saudi J Anaesth. 2017 11(4): 384-389. DOI: 10.4103/sja.SJA_84_17

[23] Kumar S., Chaudhary A.K., Singh P.K., et al. Transdermal Buprenorphine Patches for Postoperative Pain Control in Abdominal Surgery. J Clin Diagn Res. 2016; 10(6): UC05-8. DOI: 10.7860/ JCDR/2016/18152.7982. Epub 2016 Jun 1.

[24] Amra B., Rahmati B., Soltaninejad F., Feizi A. Screening Questionnaires for Obstructive Sleep Apnea: An Updated Systematic Review. Oman Med J. 2018; 33(3): 184-192. DOI: 10.5001/omj.2018.36

[25] Андреенко А.А., Долбнева Е.Л., Стамов В.И. Обеспечение проходимости верхних дыхательных путей в стационаре (второй пересмотр) [электронный документ]. Режим доступа: http:// far.org.ru/recomendation?download $=49$ \%3Adaguide. Ссылка активна на 15.12.2019. [Andreenko A.A., Dolbneva E.L., Stamov V.I. Ensuring the patency of the upper respiratory tract in the hospital (second revision) [Internet]. Available from: http://far.org.ru/ recomendation?download = 49 \%3Adaguide (accessed 15.12.2019) (In Russ)]

[26] Singh P.M., Borle A., McGavin J., et al. Comparison of the Recovery Profile between Desflurane and Sevoflurane in Patients Undergoing Bariatric Surgery - a Meta-Analysis of Randomized Controlled Trials Obes Surg. 2017; 27(11): 3031-3039. DOI: 10.1007/s11695-017-29296

[27] ASA Physical Status Classification System [Internet] Available from: https://www.asahq.org/standards-and-guidelines/asa-physicalstatus-classification-system?fbclid=IwAR2SgktgZwBO2L8aLwC-z7jM 6JmHpbdPPROKcjalDaWRS2FyjGsONg-mRio. (accessed 15.12.2019).

[28] Rasheed A.M., Amirah M.F., Abdallah M., et al. Ramsay Sedation Scale and Richmond Agitation Sedation Scale: A Cross-sectional Study Dimens Crit Care Nurs. 2019; 38(2): 90-95. DOI: 10.1097/ DCC.0000000000000346

[29] Эпштейн С.Л., Азарова Т.М., Сторожев В.Ю. и др. Общая анестезия без опиоидов в хирургии морбидного ожирения. зачем и как? Регионарная анестезия и лечение острой боли. 2016; 1(10) 47-54. DOI: 10.18821/1993-6508-2016-10-1-47-54. [Epshtein S.L., Azarova T.M., Storozhev V.Yu., et al. General anesthesia without opioids in surgery for morbid obesity. What for and how? Regional anesthesia and treatment of acute pain 2016; 1(10): 47-54. (In Russ)]

[30] Ueda K., Hussey P. Dynamic Ultrasound-Guided Short-Axis Needle Tip Navigation Technique for Facilitating Cannulation of Peripheral Veins in Obese Patients. Anesth Analg. 2017; 124(3) 831-833. DOI: 10.1213/ANE.0000000000001653

[31] Vricella L.K., Louis J.M., Mercer B.M., Bolden N. Impact of morbid obesity on epidural anesthesia complications in labor. Am. J. Obstet. Gynecol. 2011; 205(4): 370.e1-6. DOI: 10.1016/j. ajog.2011.06.085 